\title{
GaN/AlGaN back-illuminated multiple-quantum-well Schottky barrier ultraviolet photodetectors
}

\author{
A. Teke ${ }^{\text {a,b,* }}$, S. Dogan ${ }^{\text {a,c }}$, F. Yun ${ }^{\text {a }}$, M.A. Reshchikov ${ }^{\text {a }, H . ~ L e ~}{ }^{\text {a }}$, X.Q. Liu ${ }^{\text {a }}$ \\ H. Morkoç ${ }^{a}$, S.K. Zhang ${ }^{\text {d }}$, W.B. Wang ${ }^{d}$, R.R. Alfano ${ }^{\mathrm{d}}$ \\ a Department of Electrical Engineering, Virginia Commonwealth University, 601 W. Main Street, P.O. Box 843072, \\ Richmond, VA 23284-3072, USA \\ ${ }^{\mathrm{b}}$ Department of Physics, Faculty of Art \& Science, Balikesir University, 10100 Balikesir, Turkey \\ c Department of Physics, Faculty of Art \& Science, Ataturk University, 25240 Erzurum, Turkey \\ d Institute for Ultrafast Spectroscopy and Lasers and New York State Center for Advanced Technology, \\ The City College of The City University of New York, W. 138th Street and Convent Avenue, New York, NY 10031, USA
}

Received 19 December 2002; accepted 9 January 2003

\begin{abstract}
We report on characterization and operation principle of a set of GaN/AlGaN multiple-quantum-well (MQW) photovoltaic detectors. The structures were grown by molecular beam epitaxy (MBE) on $c$-plane sapphire substrates and fabricated in the back-illuminated vertical Schottky geometry. Introduction of MQWs into the active region of devices is expected to enhance the quantum efficiency due to the high absorption coefficient. A nearly flat spectral responsivity between 325 and $350 \mathrm{~nm}$ with $0.054 \mathrm{~A} / \mathrm{W}$ peak responsivity was achieved from the single-side polished backside (rough) illuminated GaN/AlGaN MQW devices. The cutoff wavelength of the MQW photodetector can be tuned by adjusting the well width, well composition and barrier height. A model has been developed to gain insight into the operation principles of MQWs photodiodes. The peak responsivity increased with decreasing barrier thickness due to enhanced tunneling of photogenerated carriers.

(C) 2003 Elsevier Science Ltd. All rights reserved.
\end{abstract}

\section{Introduction}

III-V nitride based devices are an ideal candidate for ultraviolet (UV) detection in a number of applications including early missile plume detection, flame sensing, UV astronomy, space-to-space communication and biological effects [1]. In the past decade, there have been several reports on $\mathrm{GaN}-\mathrm{AlGaN}$ based photodetectors, such as photoconductors [2], $\mathrm{p}-\mathrm{n}$ junction [3], $\mathrm{p}-\mathrm{i}-\mathrm{n}$ [4] and $\mathrm{p}-\pi-\mathrm{n}[5]$, Schottky barrier [6-8], metal-semiconductor-metal [9], metal-insulator-semiconductor [10],

\footnotetext{
${ }^{*}$ Corresponding author. Address: Department of Electrical Engineering, Virginia Commonwealth University, $601 \mathrm{~W}$. Main Street, P.O. Box 843072, Richmond, VA 23284-3072, USA. Tel.: +1-804-827-7000; fax: +1-80-827-7040.

E-mail address: ateke@mail.vcu.edu (A. Teke).
}

field-effect transistors, bipolar junction transistor [11] and avalanche photodiode [12]. Peak responsivities and corresponding quantum efficiencies scatter in a wide range depending on the device design and material qualities. Recently, Collins et al. [13] reported a peak responsivity for $\mathrm{AlGaN}$ solar-blind $\mathrm{p}-\mathrm{i}-\mathrm{n}$ photodiodes as high as $0.12 \mathrm{~A} / \mathrm{W}$ at $275 \mathrm{~nm}$ without bias, giving an external quantum efficiency of $53 \%$. This achievement was attributed to an improved material quality of $\mathrm{Al}_{0.6} \mathrm{Ga}_{0.4} \mathrm{~N}$. Design consideration and material quality are the key issues for achieving higher device performance. To date, most of the work on III-nitride UV photodetectors, inclusive of all types, has relied on bulk-like epilayers. Kipshidze et al. [14] reported GaN/ AlGaN MQW in an effort to extend the range of wavelength in LED [18]. As for infrared detectors, other III-V material systems, such as GaAs or InP based, devices containing MQWs in their active region have 
been successfully designed and demonstrated [15-17]. In a $2 \mathrm{D}$ system a high joint density of states enhances the oscillator strength, due to confinement, for upward carrier transition can enhance optical absorption, and hence lead to higher quantum efficiency in photodetectors [18]. Strong piezoelectric fields, such as those existing in GaN/AlGaN quantum wells, are also expected to improve the carrier transport. Recently, GaN/AlGaN MQWs UV photodetectors with a planar geometry with $0.03 \mathrm{~A} / \mathrm{W}$ peak responsivity have been reported [19]. In this letter we present back-illuminated vertical Schottky type MQW UV detectors and describe carrier transport along with experimental results.

\section{Growth and fabrication}

Three detectors referred hereafter as svt1171, svt1172 and svt1174 were grown by MBE using an rf-plasma as the nitrogen source, fabricated, and characterized. Fig. 1 shows the schematic of the device structure and potential profile in the growth direction. The growth was initiated by a thin layer of AlN buffer layer $(\sim 50 \mathrm{~nm})$ on top of a $c$-plane sapphire substrate following a routine thermal annealing and nitridation process. Then, a Sidoped $\mathrm{AlGaN}$ layer of $\sim 0.5 \mu \mathrm{m}$ was grown as the short wavelength cutoff region on top of the AlN buffer layer. The active layer for all three detector structures consisted of 20 periods of GaN/AlGaN MQWs with $4 \mathrm{~nm}$ well thickness. The thicknesses of barriers were 7, 5 and $3 \mathrm{~nm}$ for svt1171, svt1172 and svt1174, respectively. The growth sequence was finished by growing a thin $\mathrm{AlGaN}$ cap layer. All the layers were grown under Ga-rich conditions, inclusive of the $\mathrm{AlGaN}$ layers, which is novel [20]. Fabrication of vertical geometry Schottky diodes was initiated with the definition of mesas using reactive ion etching (RIE) in $\mathrm{BCl}_{3}$ plasma. The ohmic contact was made by electron beam and thermal evaporation of
$\mathrm{Ti} / \mathrm{Al} / \mathrm{Ti} / \mathrm{Au}$ on the conductive layer. Rapid thermal annealing for $1 \mathrm{~min}$ at $900{ }^{\circ} \mathrm{C}$ was used to yield good ohmic behavior. A Ni/Au layer composite was used for the Schottky contact in a circular pattern on the front side of the wafer with an area of $0.03 \mathrm{~mm}^{2}$.

\section{Experimental results}

The spectral responsivity for each detector structure was measured with the aid a UV-enhanced Xe arc lamp the light of which was dispersed by a monochromator. The light source was calibrated against a calibrated UVenhanced $\mathrm{Si}$ photodetector. The dispersed light was modulated with a mechanical chopper and focused on the backside of the device. The signal was amplified and analyzed by a standard lock-in technique.

Fig. 2 shows the absolute spectral response of the investigated detectors without bias. The responsivity is nearly flat between 350 and $325 \mathrm{~nm}$ for all three devices. Absorption in n-type AlGaN layer below the MQWs determines the short wavelength cutoff at 301, 305 and $312 \mathrm{~nm}$ for svt1171, svt1172 and svt1174, respectively. The $\mathrm{Al}$ content in this absorption layer was determined as, in order, $0.3,0.39$ and 0.36 by X-ray diffraction. However, the expected short cutoff wavelengths for the corresponding $\mathrm{Al}$ contents obtained by X-ray measurements are $6 \mathrm{~nm}$ longer for svt1171, and 14 (16) $\mathrm{nm}$ shorter for svt1172 (svt1174). The observation of different transition wavelengths corresponding to different Al contents from room temperature reflectance, transmittance and photoluminescence measurements is most likely due the fluctuation in $\mathrm{Al}$ compositions in $\mathrm{AlGaN}$ short wavelength filter layer of all three samples. The long wavelength cutoffs at 356,355 and $357 \mathrm{~nm}$ are determined by the ground state electron-heavy-hole absorption in the MQW for svt1171, svt1172 and svt1174, respectively. Comparing to $365 \mathrm{~nm}$ long wavelength
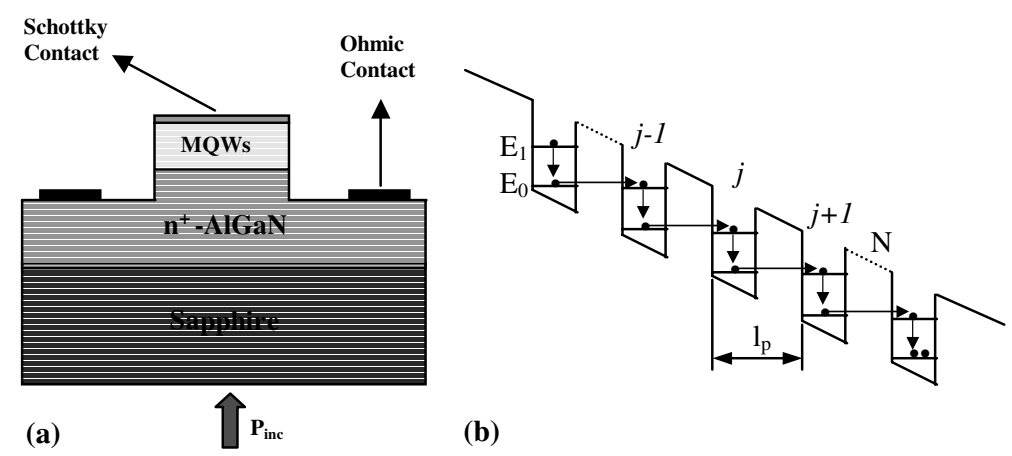

(b)

Fig. 1. (a) Schematic structure of a back-illuminated Schottky barrier multi-quantum well UV photodetector. Growth parameters are given in the text, and (b) Schematic conduction band profiles of MQW Schottky detector together with carrier transport. The meanings of the letters on the figures are given in the text. 


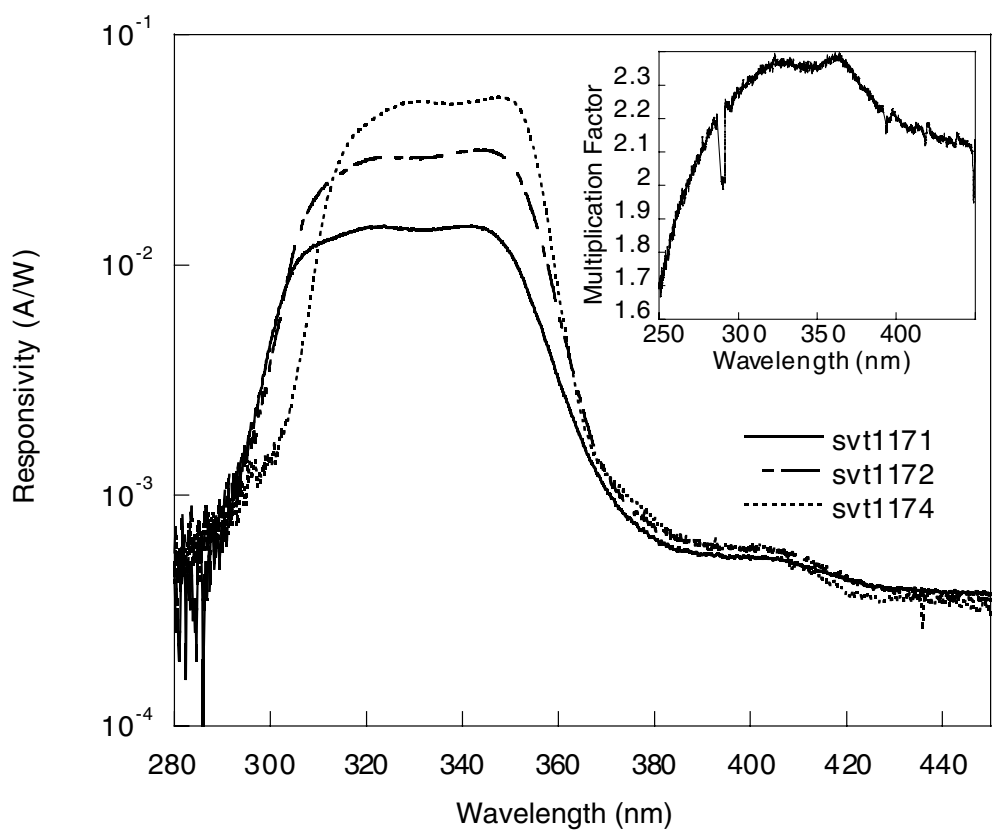

Fig. 2. Comparison of the unbiased spectral responsivity for all three MQW photodetectors. Inset: The transmission ratio between the single and double-side polished sapphire.

cutoffs of bulk type detectors, corresponding cutoffs of MQW-based detectors show about $10 \mathrm{~nm}$ blue shift. In GaN/AlGaN quantum wells, the e-hh transition energy is determined not only by quantum confinement but also by Stark-effect due to strong spontaneous and piezoelectric polarization-induced internal electric fields. The energy levels in a finite quantum well have been calculated in the absence of internal field by using structural parameters of the detectors investigated. The wavelengths corresponding to the calculated e-hh energy levels are found to be 354,353 and $355 \mathrm{~nm}$ for svt1171, svt1172 and svt1174, respectively. Due to the polarization-induced internal electric field in the MQWs, the measured long wavelength cutoff wavelengths have shown a $2 \mathrm{~nm}$ redshift.

Single-side polished back-illuminated MQWs detectors exhibited a peak responsivity value as high as 0.054 $\mathrm{A} / \mathrm{W}$ at $348 \mathrm{~nm}$ for svt1174, corresponding to $20 \%$ quantum efficiency. The particular sample has the thinnest barrier among three devices. The responsivity could be improved by using double-polished sapphire. The inset of Fig. 2 shows the transmittance ratio between single-side and double-side polished sapphire. As seen from the inset, the double-side polished sapphire transmits the light more than two times compared to singleside polished sapphire in the spectral region where devices show flat responsivity. Therefore, we would expect a peak responsivity value as high as $0.12 \mathrm{~A} / \mathrm{W}$ for svt1174 had the backside been polished. For the other detectors, svt1171 and 1172, the expected peak responsivities are about 0.035 and $0.074 \mathrm{~A} / \mathrm{W}$, respectively. Although device structures are identical, with the exception of the barrier thickness of MQW, they gave markedly varying peak responsivity values, which will be elaborated on later in the context of a model. At this point, another experimental measurement has been carried out in a support of explanation of the observed peak responsivity difference among the devices based on our model. To do so, room temperature photoluminescence (PL) spectra of both as-grown sample and their device after fabrication of ohmic and Schottky contacts have been measured by using $\mathrm{He}-\mathrm{Cd}$ laser in a standard photon counting mode. Fig. 3 shows the PL spectra taken at room temperature for all as-grown samples and detector devices. For the as-grown samples, the PL spectrum is dominated by excitonic emission of the GaN/AlGaN MQW peaked at 3.187, 3.271 and $3.358 \mathrm{eV}$ with corresponding relative PL intensities of $3.5 \times 10^{6}$, $4.2 \times 10^{6}$ and $4.9 \times 10^{6}$ for svt1171, svt1172 and svt1174, respectively. For the devices, peak energies are slightly blue-shifted and peaked at 3.214, 3.310 and $3.390 \mathrm{eV}$ with corresponding PL intensities of $9.2 \times 10^{4}, 7.9 \times 10^{4}$ and $3.9 \times 10^{4}$ for svt1171, svt1172 and svt1174, respectively. The e1-hh1 transition energies were calculated by using Eq. (1) [21] in which the red shift of the emission wavelength due to polarization-induced electric fields for as-grown and additional built-in field for detector devices in the quantum well were taken into account. 


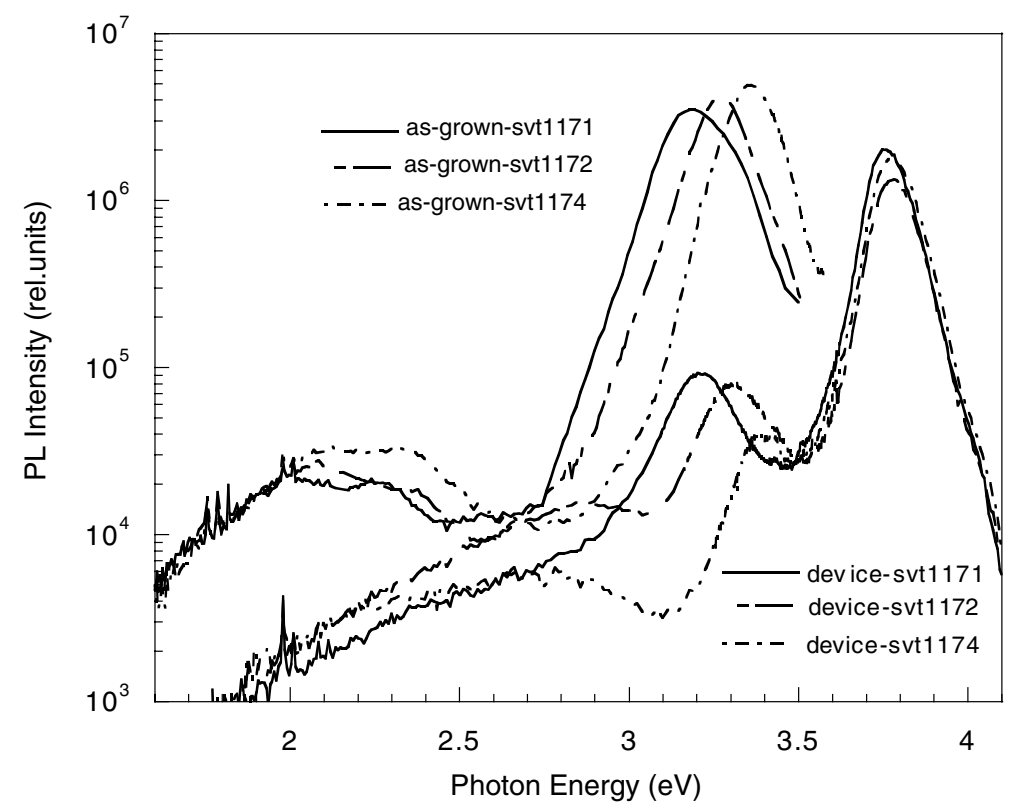

Fig. 3. Photoluminescence spectra taken at room temperature for as-grown samples and detector devices.

$E_{\mathrm{e} 1-\mathrm{hh} 1}=E_{\mathrm{g}}-l_{\mathrm{w}} F_{\mathrm{w}}+\left(\frac{9 \pi \hbar e F_{\mathrm{w}}}{8 \sqrt{2}}\right)^{2 / 3}\left(\frac{1}{m_{\mathrm{e}}^{*}}+\frac{1}{m_{\mathrm{h}}^{*}}\right)^{1 / 3}$

where $E_{\mathrm{g}}$ is the band gap of $\mathrm{GaN}, l_{\mathrm{w}}$ is the well width, $F_{\mathrm{w}}$ is the internal electric field in the well, $m_{\mathrm{e}}^{*}$ and $m_{\mathrm{h}}^{*}$ are the effective masses of electron and heavy-hole, respectively. The other parameters have their usual meaning. The calculated e1-hh1 transition energies by using the electric field in the well obtained from the model calculation, which will be discussed in the next section, are 3.217, 3.220 and $3.386 \mathrm{eV}$ for as-grown samples and 3.228, 3.231 and $3.390 \mathrm{eV}$ for detector devices, for svt1171, svt1172 and svt1174, respectively. The difference between measured and calculated peak energies can be due to uncertainty in the quantum well width. However, the observed blue shift in PL peak energies of the detector devices is an indication of the effect of built-in electric field, which partly compensates the polarization field in the quantum wells. Also as seen from the PL spectra of the detector devices, PL intensities gradually decrease compared to those of as-grown samples. Although, this decrease in part can be attributed to the enhanced absorption by and reflection from thin layer Schottky contact, the importance of this measurement is to show their relative decrease and relate them with the observed responsivities of detectors. PL intensities from devices reduce a factor of 38, 53 and 125 times compared to asgrown samples for svt1171, svt1172 and svt1174, respectively. If the relative decreases of PL intensities from the detectors are compared with one another, one can see that the PL intensity of svt1174 quenches about 3.5
(2.5) times more than that of svt1172 (svt1171). These quenching factors in PL intensities should be accompanied by an increase in the peak responsivities of the devices correspondingly. Indeed, svt1174 device shows better responsivity with a peak value of $0.054 \mathrm{~A} / \mathrm{W}$, which is a factor of about 3.5 (1.8) times higher than that of svt1172 (svt1171). Both observations (blue shift in PL peak energy and quenching in PL intensity) can be considered as a strong experimental evidence of the enhancement of escaping photogenerated carriers from the wells and contributing to the photocurrent due to enhanced tunneling probability of carriers, as the field in barrier increases, according to our model discussed in the next section. Similar observation has also been reported in literature [22-24].

The dependence of the responsivity on the incident power density was determined by using a non-focused $325 \mathrm{~nm} \mathrm{He}-\mathrm{Cd}$ laser. The optical power intensity was varied by using calibrated neutral density filters. The photocurrent exhibited a nearly linear increase with incident optical power up to $1 \mathrm{~kW} / \mathrm{m}^{2}$ for all three devices, as shown in Fig. 4.

We have also studied bias dependence of the responsivity for all three types of detectors up to $-5 \mathrm{~V}$. Since all the devices displayed similar bias characteristic, only the results from svt1174 will be presented here to avoid repetition. Fig. 5 shows the spectral responsivity taken at different bias voltages for svt1174. As seen from Fig. 5, the spectral responsivity curve does not show any significant difference as far as its shape is concerned, but the noise level increases with applied bias caused by 


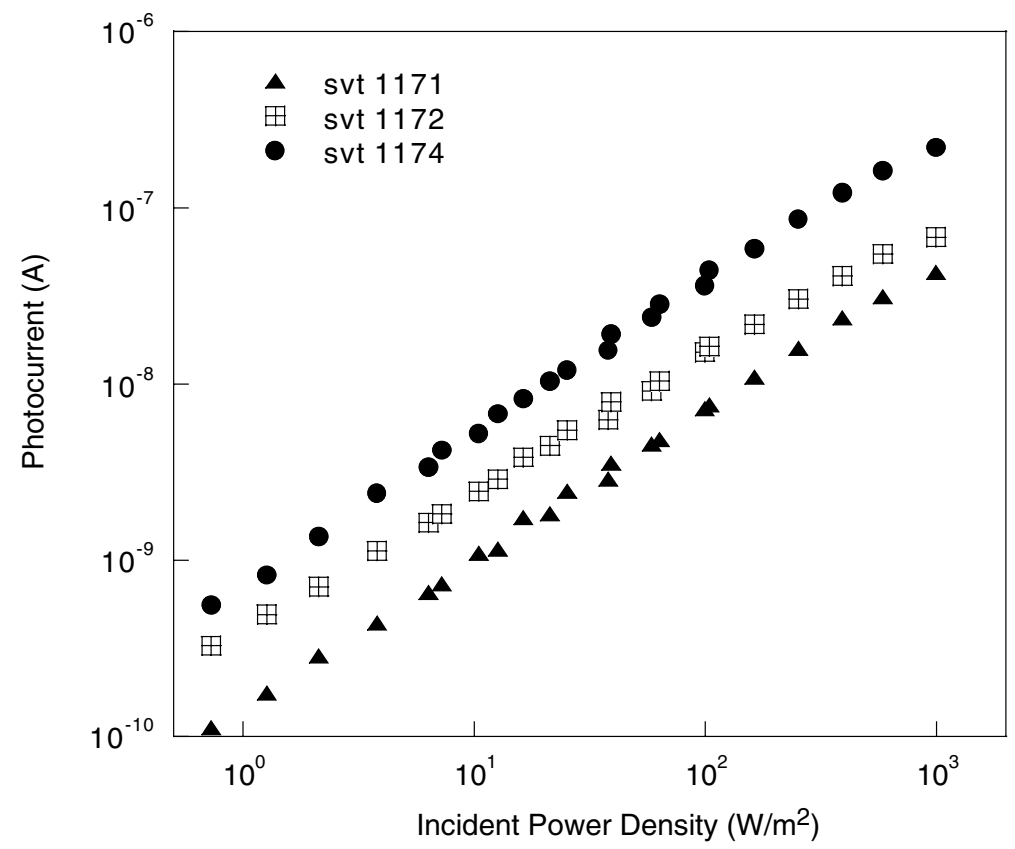

Fig. 4. Linear photocurrent dependence on the optical power density, measured with a He-Cd laser.

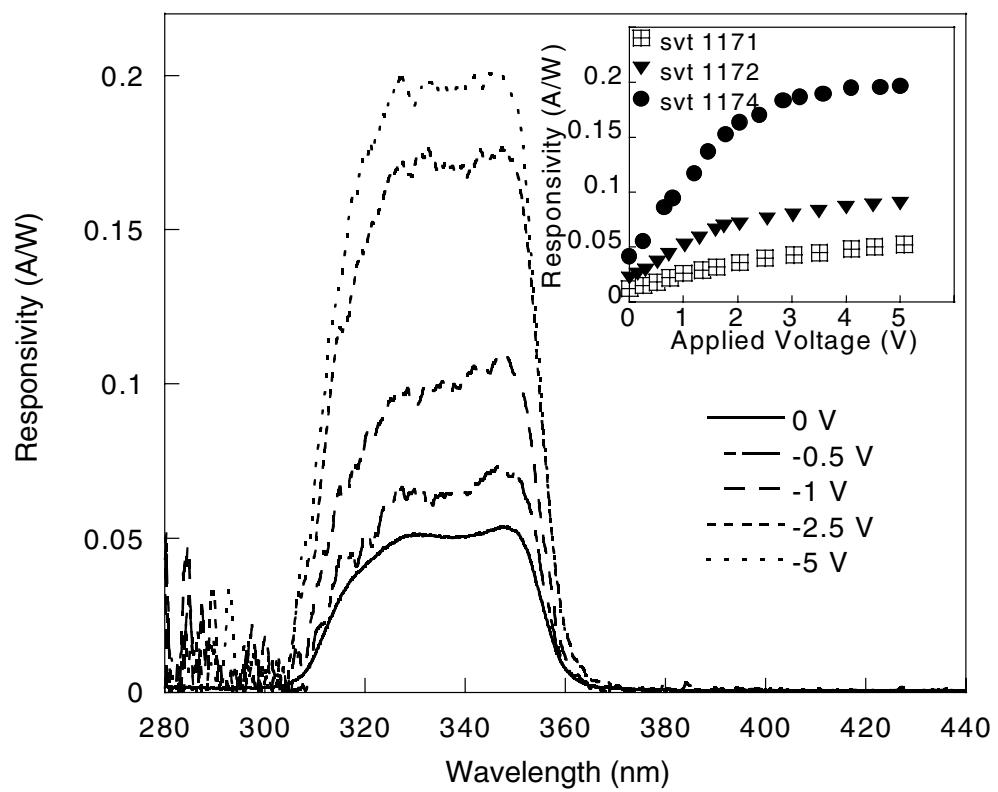

Fig. 5. Spectral responsivity at different bias voltages for the device fabricated using sample svt1174. Inset: The peak responsivity vs. the applied voltage for all three devices.

voltage source. The inset in Fig. 5 shows the peak responsivity at $350 \mathrm{~nm}$ as a function of applied bias. Below $-2 \mathrm{~V}$, the responsivity increases linearly with applied voltage. Above $-2 \mathrm{~V}$ the responsivity saturates at 0.19 $\mathrm{A} / \mathrm{W}$. The saturated responsivity values for svt1171 and svt1172 were obtained as 0.052 and $0.12 \mathrm{~A} / \mathrm{W}$, respectively. The ratio between the saturated peak responsivity and zero bias peak responsivity is about 3.6 times and the same for all devices, supporting the effect of the barrier width, which will be discussed next. 


\section{Models and discussion}

The operation mechanism of MQW photodetectors under investigation can be understood in the context of the schematic potential profile for carrier transport involved in the device operation as shown in Fig 1(b). As can be seen from the figure, in contrast to photodetectors using bulk GaN or AlGaN layers, the GaN/AlGaN MQWs are placed in the depletion region of the Schottky barrier junction. Electrons created by absorption of photons can tunnel from the ground state of the $j$ th well $(j=1,2, \ldots, N)$ into an excited state of the $(j+1)$ th well. The tunneling process is then followed by intersubband energy relaxation from the excited state to the ground sates via emitting phonons in the $(j+1)$ th quantum well. This two-step process can be repeated as many times as needed through the entire space-charge region. The sequential tunneling occurs when the condition in Eq. (2) is satisfied [17]

$E_{1}-E_{0}=\left|q F_{\text {avg }} l_{\mathrm{p}}\right|$

where $E_{0}$ and $E_{1}$ are the ground and first excited subband energies of the quantum well, $q$ is the electron charge, $F_{\text {avg }}$ is the average electric field between equivalent points in successive wells, and $l_{\mathrm{p}}$ is the spatial period of the MQW.

The calculation of the quantum well subband energies were performed in the absence of internal electric field (to be added later in the treatment), so the energy levels for electrons (holes) with respect to minimum (maximum) of the conduction (valance) band with a 70:(30) band offset ratio depend only on the well and barrier thickness, and $\mathrm{Al}$ compositions. For the well and barrier thickness growth parameters and for Al composition the values obtained from X-ray measurement were used in these calculations.

The average electric field was calculated by taking into account spontaneous and piezoelectric polarization $[22,25,26]$, built-in electric field due to the Schottky contact and applied field between the contacts. If a MQW structure is incorporated in the intrinsic region of Schottky diode, it leads to the following expression [27]

$L_{\mathrm{w}} F_{\mathrm{w}}+L_{\mathrm{b}} F_{\mathrm{b}}=-\left(V_{\mathrm{bi}}+V_{\mathrm{app}}\right)$

where $L_{\mathrm{w}}$ and $L_{\mathrm{b}}$ are the total lengths of well and barrier in the intrinsic region, $F_{\mathrm{w}}$ and $F_{\mathrm{b}}$ are the electric fields in the well and barrier, respectively. $V_{\mathrm{bi}}$ is the built-in diode voltage and $V_{\mathrm{a}}$ is the applied voltage. From the conservation of electric displacement along the growth axis, one can get the following relation

$\varepsilon_{\mathrm{w}} F_{\mathrm{w}}-\varepsilon_{\mathrm{b}} F_{\mathrm{b}}=P_{\mathrm{b}}-P_{\mathrm{w}}$

where $\varepsilon_{\mathrm{W}}\left(\varepsilon_{\mathrm{b}}\right)$ is the well (barrier) static dielectric constant, and $P_{\mathrm{w}}=P_{\mathrm{w}}^{\mathrm{sp}}+P_{\mathrm{w}}^{\mathrm{pz}}$ and $P_{\mathrm{b}}=P_{\mathrm{b}}^{\mathrm{ps}}+P_{\mathrm{b}}^{\mathrm{pz}}$ are the total polarization in the well and barrier, respectively. The superscripts sp (pz) stand for spontaneous (piezoelectric) polarization. By solving Eqs. (3) and (4) for $F_{\mathrm{w}}$ and $F_{\mathrm{b}}$, the electric field in the well can be determined as

$F_{\mathrm{w}}=\frac{L_{\mathrm{b}}\left(P_{\mathrm{b}}-P_{\mathrm{w}}\right)-\varepsilon_{\mathrm{b}}\left(V_{\mathrm{bi}}+V_{\mathrm{a}}\right)}{\varepsilon_{\mathrm{b}} L_{\mathrm{w}}+\varepsilon_{\mathrm{w}} L_{\mathrm{b}}}$

and in the barrier

$F_{\mathrm{w}}=\frac{L_{\mathrm{w}}\left(P_{\mathrm{w}}-P_{\mathrm{b}}\right)-\varepsilon_{\mathrm{w}}\left(V_{\mathrm{bi}}+V_{\mathrm{a}}\right)}{\varepsilon_{\mathrm{b}} L_{\mathrm{w}}+\varepsilon_{\mathrm{w}} L_{\mathrm{b}}}$

Then, the average electric field in MQW structure can be written as

$F_{\mathrm{avr}}=\frac{L_{\mathrm{w}} F_{\mathrm{w}}+L_{\mathrm{b}} F_{\mathrm{b}}}{L_{\mathrm{w}}+L_{\mathrm{b}}}$

The actual electric field experienced by the carriers inside the intrinsic layer is negligibly reduced from its nominal value because of the photovoltaic effect. The constants used in these calculations are given in Table 1.

In order to gain an insight whether the sequential tunneling condition is satisfied in our devices, we have graphically analyzed Eq. (1) as shown in Fig. 6. In this

Table 1

Lattice constants, spontaneous polarization, piezoelectric, dielectric and elastic constants of $\mathrm{AlN}, \mathrm{GaN}$ and $\mathrm{Al}_{x} \mathrm{Ga}_{1-\mathrm{x}} \mathrm{N}$ used in the calculation

\begin{tabular}{lccl}
\hline Parameters & AlN & GaN & $\mathrm{Al}_{x} \mathrm{Ga}_{1-\mathrm{x}} \mathrm{N}$ \\
\hline$a_{0}(\AA)$ & 3.112 & 3.189 & $-0.077 x+3.189$ \\
$e_{33}\left(\mathrm{C} / \mathrm{m}^{2}\right)$ & 1.46 & 0.73 & {$\left[e_{33}(\mathrm{AlN})-e_{33}(\mathrm{GaN})\right] x+e_{33}(\mathrm{GaN})$} \\
$e_{31}\left(\mathrm{C} / \mathrm{m}^{2}\right)$ & -0.60 & -0.49 & {$\left[e_{31}(\mathrm{AlN})-e_{31}(\mathrm{GaN})\right] x+e_{31}(\mathrm{GaN})$} \\
$C_{13}(\mathrm{GPa})$ & 108 & 103 & $5 x+103$ \\
$C_{33}(\mathrm{GPa})$ & 373 & 405 & $-32 x+405$ \\
$\varepsilon_{33}$ & 10.1 & 10.4 & $-0.3 x+10.4$ \\
$P^{\mathrm{sp}}\left(\mathrm{C} / \mathrm{m}^{2}\right)$ & -0.081 & -0.029 & $-0.052 x-0.029$ \\
$P^{\mathrm{pz}}\left(\mathrm{C} / \mathrm{m}^{2}\right)$ & - & - & $2\left[\frac{a_{\mathrm{s}}(x)-a(x)}{a(x)}\right]\left[e_{31}(x)-e_{33}(x) \frac{C_{13}(x)}{C_{33}(x)}\right]$ \\
\hline
\end{tabular}

$x$ is the $\mathrm{Al}$ composition in $\mathrm{Al}_{x} \mathrm{Ga}_{1-x} \mathrm{~N}, a_{\mathrm{s}}$ is lattice constant of buffer layer and $a$ is lattice constant of epilayers. These parameters are taken from reference [23]. 


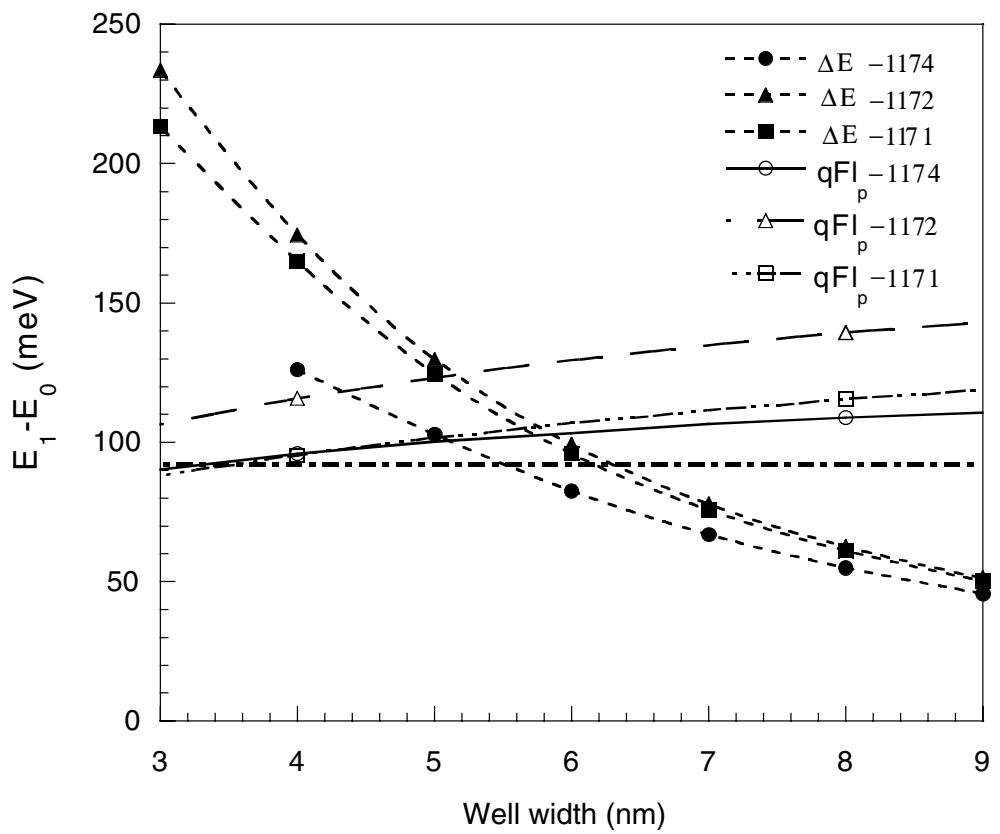

Fig. 6. Graphical solution of the equation $\Delta E=\left|q F_{\text {avr }} l_{\mathrm{p}}\right|$. Filled tri-angles, squares and circles show the calculated difference $\Delta E=E_{1}-E_{0}$ (the dashed lines on data are interpolating curve fit for eye trace) and empty tri-angles, squares and circles are $\left|q F_{\text {avr }} l_{\mathrm{p}}\right|$ for svt1171, svt1172 and svt1174, respectively. The horizontal line is drawn to mark the $\Delta E=92$ meV boundary.

figure, we present the calculated energy separation of the ground and first excited states, $\Delta E=E_{1}-E_{0}$ and the value of $\left|q F_{\text {avr }} l_{\mathrm{p}}\right|$ for all three detectors as a function of well width for zero applied field. Since structures of the devices studied in this work have different barrier widths and $\mathrm{Al}$ compositions, both in the barrier of intrinsic MQW region and $\mathrm{AlGaN}$ short wavelength cutoff layer, the figure also shows the dependence of $\Delta E$ and $\left|q F_{\text {avr }} l_{\mathrm{p}}\right|$ on these parameters. The horizontal line at $\Delta E=92$ $\mathrm{meV}$ is also drawn to mark the optical phonon boundary for intersubband energy relaxation. As clearly seen from the figure, while $\Delta E$ decreases with increasing well width, it increases with increasing barrier width and $\mathrm{Al}$ composition. At $l_{\mathrm{w}}=4 \mathrm{~nm}$, which is the quantum well width for all three devices, the calculated energy separations between the first excited and ground states in the conduction band are 165, 175 and $126 \mathrm{meV}$ for svt1171, svt1172 and svt1174, respectively. Although the resonant tunneling condition, even taking into account the broadening due to electron-electron, electron-phonon, electron-impurity scattering and potential fluctuation (on the order of $6 \mathrm{meV}$ ), has not been satisfied for any of the samples, comparison of devices in terms of their growth parameters can be used for interpretation and analyzing the observed experimental results. Firstly, for all three samples, $\Delta E$ is greater than $\left|q F_{\mathrm{avr}} l_{\mathrm{p}}\right|$. At $l_{\mathrm{w}}=4$ $\mathrm{nm}$, the differences between $\Delta E$ and $\left|q F_{\mathrm{avr}} l_{\mathrm{p}}\right|$ are 70,59 and $30 \mathrm{meV}$ for svt1171, svt1172 and svt1174 with barrier widths, $l_{\mathrm{b}}$, of 7, 5 and $3 \mathrm{~nm}$, respectively. Thus, the device having a minimal difference and thinnest barrier (svt1174) shows the best responsivity with a peak value of $0.054 \mathrm{~A} / \mathrm{W}$, which is a factor of about two and four times higher than that of svt1172 and svt1171, respectively. As also clearly be noticed, better performance of svt1172 over svt1171 is also determined by these two parameters.

Since the average field in the intrinsic region is linearly dependent on the applied voltage $\left|q F_{\text {avr }} l_{\mathrm{p}}\right|$ also increases linearly with $V_{\mathrm{a}}$, which is not shown in the figure for clarity. As observed experimentally, the peak responsivity increases with increasing applied reverse voltage due to the increased average electric field across the increased depletion region. Thus, a combination of increased band bending and the depletion region encompassing more of the quantum wells enables more efficient carrier collection at the terminals, leading to a higher photocurrent across the external circuit. It should be noted that the difference between $\Delta E$ and $\left|q F_{\text {avr }} l_{\mathrm{p}}\right|$ decreases with increasing applied bias, resulting in enhancement of the tunneling process. However, no resonant tunneling was observed in the measured voltage range. The non-uniformity of the device parameters, such as well width, barrier width, alloy composition and electric field in each individual period could hinder the observation of this effect. At higher applied bias voltages, the measured responsivity exhibited 
photoconductive gain, probably originating from defects at the surface and/or within the depletion layer. Then, it tends to saturate due to sweep-out effect [28]. Comparing the ratio of the zero bias and the saturated peak responsivity for all three devices, almost a constant value is found, indicating the effect of the barrier thickness.

In conclusion, we presented vertical geometry Schottky type GaN/AlGaN MQW UV photodetectors. Three device structures having different barrier thickness were designed and investigated. A maximum peak responsivity achieved in our detectors is $0.054 \mathrm{~A} / \mathrm{W}$ in a backilluminated geometry with rough sapphire back side. The spectral responsivity window can be tuned to shorter wavelengths by variation the quantum well thickness and increasing the Al mole fraction. It has been shown that the photocurrent was limited by tunneling through the barriers, which upon further optimization can lead to improved device performance. The optimization criteria are: (i) for a given $\mathrm{Al}$ composition and hence given band offset, the lower limit on the well width is imposed by the requirement of having at least two subbands inside the well, on the other hand upper limit should be set to a value to facilitate an efficient intersubband energy relaxation through emission of optical phonons $(\Delta E \geqslant$ $\left.\hbar \omega_{L O}\right)$, where $\hbar \omega_{L O} \approx 92 \mathrm{meV}$ is the optical phonon energy for GaN; (ii) the thickness of the barrier should be neither very large because of a low tunneling probability, nor too small due to low absorption efficiency at shorter wavelengths; (iii) to provide resonant tunneling, the average electric field should be set to a value close to the first excited and ground states energy separation (to do so, for a given $\mathrm{Al}$ content, thickness of well and barrier and their ratio, and period of superlattices and number of periods have to be chosen consistently by taking the above criteria into account); (iv) quality of the layers should also be considered in designing better performance MQW UV photodetectors; (v) to obtain a complete picture in understanding the device operation and comparison with the experiment results, calculation of the current densities through tunneling and thermionic emission needs to be included in the proposed model. Further determination of other parameters, such as bandwidth, and detectivity together with optimization of device structure is underway.

\section{Acknowledgements}

This collaborative research between VCU and CCNY is supported by AFOSR grant \# F49620-01-10188. We thank Dr. Todd Steiner for useful discussion and advise. The work at VCU also benefited from other grants by AFOSR, ONR and NSF. The work at CCNY was partially supported by New York State Technology Foundation.

\section{References}

[1] Morkoç H, Di Carlo A, Cingolani R. Solid-State Electron 2002;46:157.

[2] Kung P, Zhang X, Walker D, Saxler A, Piotrowsky J, Rogalski J, et al. Appl Phys Lett 1995;67:3792.

[3] Monroy E, Munoz E, Sanchez FJ, Calle F, Calleja E, Beaumont B, et al. Semicond Sci Technol 1998;13:1042.

[4] Sandvik P, Mi K, Shahedipour F, McClintock R, Yasan A, Kung P, et al. J Cryst Growth 2001;231(3):366.

[5] Osinsky A, Gangopadhyay S, Gaska R, Williams B, Khan AA, Kuksenkow D, et al. Appl Phys Lett 1997;71(16):2334.

[6] Katz O, Garber V, Meyler B, Bahir G, Salzman J. Appl Phys Lett 2001;79(10):1417.

[7] Kuryatkov VV, Kipshidze GD, Nikishin SA, Deelman PW, Temkin H. Phys Stat Sol (a) 2001;188(1):317.

[8] Biyikli N, Kartaloglu T, Aytur O, Kimukin I, Ozbay E. Appl Phys Lett 2001;70(17):2838.

[9] Pau JL, Monroy E, Sanchez-Garcia MA, Calleja E, Munoz E. Mater Sci Eng B-Solid 2002;93(1-3):159.

[10] Monray E, Calle F, Pau JL, Munoz E, Omnes F, Beaumont B, et al. J Cryst Growth 2001;270:541.

[11] Moullet R, Hirano A, Iwaya M, Detchprohm T, Amona H, Akasaki I. Jpn J Appl Phys 2001;40(Part 2):L498.

[12] Carrano JC, Lambert DJH, Eiting CJ, Collins CJ, Li T, Wang S, et al. Appl Phys Lett 2000;76(7):924.

[13] Collins CJ, Chowdhury U, Wong MM, Yang B, Beck AL, Dupuis RD, et al. Electron Lett 2002;38(15):824.

[14] Kipshidze G, Kuryatkov V, Borisov B, Nikishin S, Holtz M, Chu SNG, et al. Phys Stat Sol (a) 2002;192(2):286.

[15] Shimada Y, Hirakawa K. Jpn J Appl Phys 1998;37(part 1): 1421.

[16] Raisky OY, Wang WB, Alfano RR, Reynolds Jr CL. Appl Phys Lett 2001;79(3):430.

[17] Raisky OY, Wang WB, Alfano RR, Reynolds Jr CL, Stampone DV, Focht MW. Appl Phys Lett 1999;74(1):129.

[18] Bhattacharya P. Semiconductor optoelectronics devices. Prentice-Hall; 1994.

[19] Zhang SK, Wang WB, Yun F, He L, Morkoç H, Zhou X, et al. Appl Phys Lett 2002;81(24):4628.

[20] He L, Reshchikov MA, Yun F, Huang D, King T, Morkoç H. Appl Phys Lett 2002;81:2178.

[21] Cingolani R, Botchkarev A, Tang H, Morkoç H, Traetta G, Coli G, et al. Phys Rev B 2000;61(4):2711.

[22] Leroux M, Grandjean N, Massies J, Gil B, Lefebvre P, Bigenwald P. Phys Rev B 1999;60(3):1496.

[23] Sanchez-Rojas JL, Sacedon A, Calle F, Calleja E, Munoz E. Appl Phys Lett 1994;65(17):2214.

[24] Horikoshi Y, Fischer A, Ploog K. Phys Rev B 1985; 31(12):7895.

[25] Ambacher O, Foutz B, Smart J, Shealy JR, Weimann NG, Chu K, et al. J Appl Phys 2000;87(1):334.

[26] Fiorentini V, Bernardini F, Della Sala F, Di Carlo A, Lugli P. Phys Rev B 1999;60(2):8849.

[27] Pabla AS, Sanchez-Rojas JL, Woodhead J, Grey R, David JPR, Rees GJ, et al. Appl Phys Lett 1993;63(6):752.

[28] Razeghi M, Rogalski A. J Appl Phys 1996;79(10):7433. 